\title{
Concentraciones plasmáticas de piperacilina/tazobactam en pacientes pediátricos críticos sometidos a ECMO. Análisis preliminar
}

\section{Plasmatic concentracion of piperacillin/tazobactam in pediatric patients on ECMO support. Preliminary analysis}

Brenda Zylbersztajn', Giannina Izquierdo C. ${ }^{1}$, Daniel Navea M. ${ }^{1}$, Juan Pablo Torres T. ${ }^{1}$ y Cristián Valverde G. ${ }^{1}$

Clínica Las Condes.

Los autores declaran no tener conflicto de interés.

Fuente de financiamiento: PIDA-CLC 2016-007. Clínica Las Condes. Santiago, Chile.

Recibido: 27 de noviembre de 2019 / Aceptado: 22 de mayo de 2020

\section{Resumen}

Introducción: Se desconocen las alteraciones farmacocinéticas de piperacilina/tazobactam (PT) en pacientes pediátricos que requieren de membrana de oxigenación extracorpórea (ECMO) y cómo dosificar adecuadamente dicho antimicrobiano. Objetivo: Describir las concentraciones plasmáticas (CP)y evaluar el cumplimiento del objetivo farmacocinético/famacodinámico de piperacilina en pacientes pediátricos en soporte con ECMO. Métodos: Presentamos tres pacientes pediátricos en tratamiento con $\mathrm{PT}$ que requirieran de ECMO en los que se midieron CP de piperacilina en la mitad del intervalo de dosificación mediante cromatografía liquida de alta resolución. Resultados: Las CP fueron $51,7-14,1$ y $6,5 \mu \mathrm{g} / \mathrm{mL}$ para los pacientes A, B y C, respectivamente. Sólo se alcanzaron CP adecuadas en un paciente. Conclusión: Estos resultados preliminares sugieren que la disponibilidad de CP de piperacilina podría optimizar el cumplimiento de los objetivos farmacocinéticos/farmacodinámicos en pacientes pediátricos en soporte con ECMO.

Palabras clave: Membrana de oxigenación extracorpórea; piperacilina tazobactam; pediatría; farmacocinética.

\section{Introducción}

L a oxigenación por membrana extracorpórea (ECMO) se ha convertido en un medio esencial de apoyo para la insuficiencia cardio-respiratoria grave en pacientes con enfermedades críticas $^{1}$. Algunos de ellos cursan con infecciones graves que requieren una terapia antimicrobiana efectiva. La enfermedad crítica y el soporte en ECMO induce cambios significativos en la farmacocinética (FC) de los antimicrobianos, dando lugar a concentraciones plasmáticas (CPs)

\begin{abstract}
Background: Pharmacokinetics and optimal dosing of piperacillin tazobactam (PT) have not been well studied in pediatric patients undergoing extracorporeal oxygenation membrane (ECMO). Aim: To describe piperacillin plasmatic concentration and evaluate achievement of pharmaccokinetic/pharmacodinamic objective in patients on ECMO support. Method: We report three pediatric patients admitted to the Pediatric Intensive Care Unit, treated with PT undergoing ECMO. Plasmatic concentrations of piperacillin were obtained in the middle of the dosing interval using high performance liquid chromatography. Results: Plasmatic concentrations were $51,7-14,1$ and $6,5 \mu \mathrm{g} / \mathrm{mL}$ for patient A, B and C respectively. Only one patient reached adequate concentrations. Conclusion: These preliminary results suggest that availability of plasmatic concentrations of piperacillin could optimize the achievement of pharmacokinetic/pharmacodynamic objectives in pediatric patients on ECMO support.

Keywords: Extracorporeal oxygenation membrane; piperacillin tazobactam; pediatrics; pharmacokinetics.
\end{abstract}

insuficientes para tratar patógenos susceptibles ${ }^{2,3}$.

Piperacilina/tazobactam (PT), es un antimicrobiano de amplio espectro, comúnmente indicado para tratamiento empírico en pacientes hospitalizados pediátricos, incluso en Unidad de Cuidados Intensivos ${ }^{4}$. Su efectividad posee una clara relación con el tiempo que se mantiene la CP por encima de la concentración inhibitoria mínima (CIM) ${ }^{5,6}$. Se desconocen las alteraciones FC que puede presentar este antimicrobiano en pacientes pediátricos conectados a ECMO y cómo optimizar la dosificación. 


\section{Metodología}

Los pacientes ingresados entre los años 2016 y 2019 al centro de pacientes críticos pediátricos de Clínica las Condes, que requirieran de ECMO y con tratamiento con $\mathrm{PT}$, fueron incluidos en un ensayo clínico FC/farmacodinámico (FD) de determinación de CPs de meropenem, vancomicina y PT, aprobado por el Comité de Ética de la institución. Recibieron una dosis de PT de $300 \mathrm{mg} /$ $\mathrm{kg} /$ día (en base a piperacilina) cada $8 \mathrm{~h}$. Se infundió el antimicrobiano en tres horas y se obtuvo CPs una hora post infusión. Se consideró adecuado un parámetro $\mathrm{FC} /$ FD de al menos 50\% del tiempo del intervalo sobre la CIM (t50 > CIM), considerando el punto de corte de $16 \mu \mathrm{g} / \mathrm{mL}$ para Pseudomonas aeruginosa ${ }^{7}$. Las muestras para cuantificar CPs fueron obtenidas luego de $24 \mathrm{~h}$ del inicio del régimen de dosificación y la concentración de piperacilina fue determinada por cromatografía liquida de alta resolución Agilent Infinity 1260 con detector UV.

\section{Resultados (Tabla 1)}

\section{Paciente A}

Sexo masculino, de 9 meses, con diagnóstico de neumonía por influenza conectado a ECMO venoarterial.

\section{Paciente B}

Sexo femenino, de 2 años, conectada a ECMO veno venoso (VV), con diagnóstico de neumonía por adenovirus.

\section{Paciente C}

Sexo masculino, de 11 años, conectado a ECMO VV post cirugía de estenosis bronquial.

Los t50 de piperacilina fueron 51,$7 ; 14,1$ y $6,5 \mu \mathrm{g} /$ $\mathrm{mL}$, el balance hídrico en $24 \mathrm{~h}$ fue $-1.000 ; 750$ y 1.170 $\mathrm{mL}$ y la proteína $\mathrm{C}$ reactiva fue $8 ; 56$ y $155 \mathrm{mg} / \mathrm{L}$ para los pacientes A, B y C, respectivamente.

Albuminemia, creatininemia y nitrógeno ureico en sangre fueron normales en los tres pacientes. Ninguno de ellos requirió terapia de reemplazo renal.

Las dosis del antimicrobiano no fueron ajustadas debido al retiro del soporte ECMO posterior a la toma de muestra o cambio de indicación del antiinfeccioso por deterioro clínico del paciente.

\section{Discusión}

Con excepción de algunos datos en poblaciones neonatales $^{8}$, no han sido bien descritas las alteraciones FC de los $\beta$-lactámicos durante el soporte en ECMO, con la consecuente falta de certeza sobre cómo optimizar el régimen de dosificación para una exposición adecuada.

Las CPs de piperacilina obtenidas en nuestros pacientes mostraron una gran dispersión inter-individual, alcanzando el objetivo FC/FD en el paciente A, pero no en los pacientes B y C. Analizando el balance hídrico, los pacientes $\mathrm{B}$ y $\mathrm{C}$, tuvieron un balance positivo 755 y $1.170 \mathrm{~mL}$ en $24 \mathrm{~h}$, respectivamente. En paralelo, las CPs fueron más bajas que en el paciente A, quien tuvo un balance hídrico negativo $1.000 \mathrm{~mL}$ en $24 \mathrm{~h}$. Dichos valores podrían explicar la variación de las CPs obtenidas debido a un potencial aumento del VD.

Actualmente no existen estudios FC de PT en ECMO en población pediátrica. En población adulta, Donadello y cols., realizaron un estudio sobre el uso de $\beta$-lactámicos en ECMO. Sólo $40 \%$ de los adultos en tratamiento con PT alcanzaron la exposición objetivo para el tratamiento de $P$. aeruginosa cuando recibieron una dosis de $4 \mathrm{~g}$ cada $6 \mathrm{~h}^{9}$.

Cies y cols., estudiaron la farmacocinética poblacional de PT en pacientes pediátricos críticamente enfermos. Los regímenes de dosificación que lograron el objetivo (punto de corte de $16 \mu \mathrm{g} / \mathrm{mL}$ para $P$. aeruginosa) fueron $100 \mathrm{mg} / \mathrm{kg}$ cada $6 \mathrm{~h}$ administrados en $3 \mathrm{~h}$ y $400 \mathrm{mg} / \mathrm{kg}$ administrados en forma infusión continua ${ }^{4}$, por lo que sería de preferencia utilizar estos esquemas de dosificación, para asegurar concentraciones plasmáticas adecuadas.

Nuestros resultados preliminares destacan la importancia de contar con la determinación de CPs de PT para poder optimizar la dosificación del antimicrobiano en pacientes pediátricos con soporte de ECMO.

Agradecimientos. Los autores quisieran agradecer a los doctores Jaime Cordero, Roberto Dalmazzo y Rodrigo Díaz, EU Christian Fajardo, QF Jorge Morales y al equipo de enfermería del Centro de Paciente Critico Pediátrico de Clínica Las Condes por su cooperación y apoyo.

Tabla 1. Resultados bioquímicos y balance hídrico de los pacientes

\begin{tabular}{lccc}
\hline & Paciente A & Paciente B & Paciente C \\
Albumina $(\mathrm{g} / \mathrm{L})$ & 3,2 & 3,6 & 3,5 \\
$\mathrm{NU}(\mathrm{mg} / \mathrm{dL})$ & 4 & 10,8 & 12,5 \\
Creatinina sérica $(\mathrm{mg} / \mathrm{dL})$ & 0,14 & 0,21 & 0,51 \\
$\mathrm{PCR}(\mathrm{mg} / \mathrm{L})$ & 8 & 56 & 155 \\
$\mathrm{CP} P(\mu \mathrm{g} / \mathrm{mL})$ & 51,7 & 14,1 & 6,5 \\
Balance hídrico $24 \mathrm{~h}(\mathrm{~mL})$ & -1000 & 755 & 1170 \\
\hline NU: nitrógeno ureico; PCR: proteína C reactiva; CP: concentración plasmática; P: piperacilina.
\end{tabular}




\section{Referencias bibliográficas}

1.- Fraser J F, Shekar K, Diab S, Foley S R, McDonald C I, Passmore M, et al. ECMO-the clinician's view. ISBT Sci Ser 2012; 7: 82-8. doi:10.1111/j.1751-2824.2012.01560.x.

2.- Taccone F S, Hites M, Beumier M, Scolletta S, Jacobs F. Appropriate antibiotic dosage levels in the treatment of severe sepsis and septic shock. Curr Infect Dis Rep 2011; 13: 406-15. doi: 10.1007/s11908-011-0203-y.

3.- Shekar K, Fraser J F, Smith M T, Roberts J A. Pharmacokinetic changes in patients receiving extracorporeal membrane oxygenation. J Crit Care 2012; 27: e9-18. doi: 10.1016/j. jcrc.2012.02.013.

4.- Cies J J, Shankar V, Schlichting C, Kuti J L.
Population pharmacokinetics of piperacillin/ tazobactam in critically ill young children. Pediatr Infect Dis J 2014; 33 (2): 168-73. doi: 10.1097/INF.0b013e3182a743c7.

5.- Drusano G L. Prevention of resistance: A goal for dose selection for antimicrobial agents. Clin Infect Dis. 2003; 36: 42-50. doi:10.1086/344653.

6.- Tam V H, Louie A, Lomaestro B M, Drusano G L. Integration of population pharmacokinetics, a pharmacodynamic target, and microbiologic surveillance data to generate a rational empiric dosing strategy for cefepime against Pseudomonas aeruginosa. Pharmacotherapy. 2003; 23: 291-5. doi:10.1592/ phco.23.3.291.32110.

7.- The European Committee on Antimicrobial
Susceptibility Testing. Breakpoint tables for interpretation of MICs and zone diameters. Version 9.0, 2019. http://www. eucast.org

8.- Ahsman M J, Wildschut E D, Tibboel D, Mathot R A. Microanalysis of $\beta$-lactam antibiotics and vancomycin in plasma for pharmacokinetic studies in neonates. Antimicrob Agents Chemother 2009; 53: 75-80. doi: 10.1128/AAC.00636-08.

9.- Donadello K, Antonucci E, Cristallini S, Roberts J A, Beumier M, Scolletta S, et al. $\beta$-Lactam pharmacokinetics during extracorporeal membrane oxygenation therapy: A case-control study. Int J Antimicrob Agents. 2015; 45 (3): 278-82. doi: 10.1016/j. ijantimicag.2014.11.005. 